\title{
Self-organization at air-water interfaces emerging from Marangoni and elastocapillary effects directed by amphiphile filament connections
}

\author{
Mitch Winkens, Peter A. Korevaar* \\ Institute for Molecules and Materials, Radboud University, The Netherlands \\ e-mail: p.korevaar@science.ru.nl
}

\begin{abstract}
Marangoni and elastocapillary effects are wellknown as driving forces in the self-organization of floating objects at air-water interfaces. The release of surface active compounds generates Marangoni flows that cause repulsion, whereas capillary forces drive attraction. Typically, these interactions are non-directional and mechanisms to establish directional connections between the self-organizing elements are lacking. In this work, we unravel the mechanisms involved in the selforganization of a linear amphiphile into millimeter-long filaments that form connections between floating droplets. First, we show how the

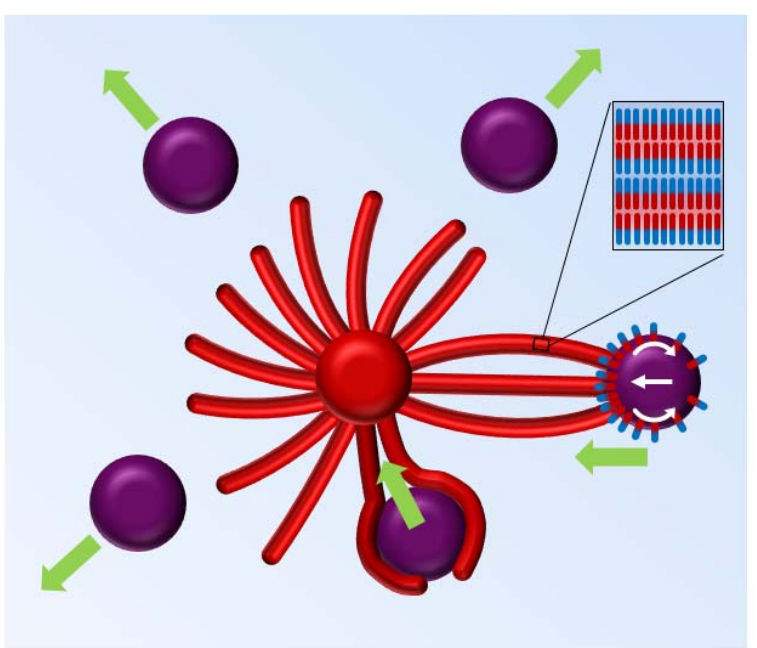
release of the amphiphile tetra(ethylene glycol) monododecyl ether from a floating source droplet onto the air-water interface generates a Marangoni flow. This flow extrudes self-assembled amphiphile filaments which grow from the source droplet, and concomitantly repels floating droplets in the surroundings. A hydrophobic drain droplet that depletes the amphiphiles from the air-water interface directs the Marangoni flow and thereby the growing filaments. We show how these filaments, upon tethering to the drain, potentially facilitate internal Marangoni convection and elastocapillary effects, which attract the drain back towards the source droplet. Furthermore, this concept establishes connections that are selective to the composition of the drain droplets - which influences the rate at which they deplete the amphiphile - such that repulsive and attractive forces can be balanced. Thereby, we provide a novel method through which directional attraction can be established in synthetic self-organizing systems, and advance our understanding of how complexity arises from simple building blocks.
\end{abstract}

\section{Introduction}

Self-organization is pivotal to the formation of functional structures in life - varying from the cytoskeleton that controls cell division, ${ }^{1,2}$ to slime mold networks that optimize nutrient acquisition, ${ }^{3}$ and interconnected neurons that establish the brain function. ${ }^{4}$ Synthetic systems can help us to understand how such complex phenomena can emerge from the interactions between a minimalistic set of building blocks. ${ }^{5}$ At the same time, control over self-organization provides paradigms to create novel types of matter that spontaneously generate functional patterns and structures. ${ }^{6-9}$ Pattern generation 
from initially homogeneous solutions has been established in reaction-diffusion based systems such as the Belousov-Zhabotinsky reaction ${ }^{10,11}$ and the thio-urea reaction, ${ }^{12}$ as well as via Rayleigh-Bénard convection that employs minute substrate-product buoyancy differences in an enzyme-mediated reaction network. ${ }^{13}$ Self-organization of complex microstructures has been demonstrated in inorganic precipitation reactions, directed by gradients that emerge around the growing structures. ${ }^{14,15}$ Cytoskeletal motors and microtubules together constitute in vitro active fluids that organize into structural flow patterns. ${ }^{16}$

Furthermore, self-organization can emerge from the collective behavior of elements that move individually. Droplet-based systems self-organize into swarms that form dynamic structures, ${ }^{17-19}$ display motion resembling predatory behavior, ${ }^{20,21}$ transfer information, ${ }^{22}$ or solve mazes. ${ }^{23,24}$ The driving forces for such self-organization processes result from interactions between the elements and the surrounding fluid or substrate. The Marangoni effect drives a liquid to flow from regions with low surface tension to those with high surface tension. ${ }^{25-27}$ Upon release of a surface active compound, floating objects can initiate Marangoni flows in the surrounding fluid, such that the objects repel each other and thereby self-organize. In case of a liquid droplet, a surfactant that is absorbed or generated at the droplet boundary generates a difference in surface tension between the front and the rear of the droplet. This imbalance results in an internal Marangoni flow, which propels the droplet towards the side with the highest surfactant concentration. ${ }^{25,26,28}$

Alternatively, self-organizing elements can interact via capillary effects, which involve the deformation of a liquid interface caused by objects in contact with it. ${ }^{29}$ Capillary forces have been demonstrated to deform elastic microstructures, ${ }^{30,31}$ even causing them to collapse into hierarchical assemblies. ${ }^{32,33}$ Furthermore, floating objects with lower density than the supporting liquid will be surrounded by either an upward or downward meniscus, if wetting is favorable or unfavorable, respectively, ${ }^{34,35}$ whereas floating objects of higher density are always surrounded by a downward meniscus. ${ }^{36}$ To minimize the air-water interfacial area, floating objects with similar menisci attract one another, which enables self-organization. . $^{18,37,38}$

Typically, these self-organization phenomena rely on the emergence of gradients, e.g. in concentration, surface tension or surface curvature. ${ }^{34,39,40}$ However, the spreading of these gradients is non-directional, and mechanisms by which self-organization is mediated through specific, directional connections between the elements - where some elements attract each other while others are repelled are seldom reported. Recently, our research group published a droplet-based system with the ability to self-organize into a spatially coordinated network. ${ }^{41}$ Crucially, the positioning of elements in this system is regulated through millimeter-long filaments, which grow from a droplet of the amphiphile tetra(ethylene glycol) monododecyl ether $\left(\mathrm{C}_{12} \mathrm{E}_{4}\right)$ that is deposited at an air-water interface. The amphiphile self-assembles into a lamellar phase of closely packed bilayers at the boundary of the droplet; the spaces in between these bilayers take up water and the resulting osmotic pressure forces the bilayers to buckle and form multilamellar filaments - known as 'myelin figures' in literature - which progress over the air-water interface (Figure 1a). ${ }^{42,43}$ Furthermore, the source droplet releases free $\mathrm{C}_{12} \mathrm{E}_{4}$ molecules to the air-water interface. This interfacial $\mathrm{C}_{12} \mathrm{E}_{4}$ is slowly depleted due to its migration into the underlying aqueous phase, which drives a Marangoni flow that helps to extrude the growing filaments from the source. ${ }^{44}$ When a drain droplet of a hydrophobic liquid is deposited as well, it also depletes $\mathrm{C}_{12} \mathrm{E}_{4}$ from the air-water interface. The resulting surface tension gradient generates a Marangoni flow that directs filaments towards the drain. Upon arrival of the filaments, the drain is drawn back towards the source (Figure 1a, b).

In this paper, we further unravel the mechanisms involved in the self-organization of the floating droplets and filaments, which relies on a balance between the repulsive Marangoni flow and the tethering of the filaments to the drain. Furthermore, we demonstrate how connectivity established by self-assembling filaments mediates directional control over the forces that position the elements in self- 
organization. To this end, we first assess the Marangoni flow in the aqueous solution (Figure 1b). Next, we investigate the role of the internal Marangoni flow (Figure 1c) and capillary effects (Figure 1d) in the attraction of drain droplets. Finally, the obtained insights are exploited in a selective self-organizing system where drain droplets, based on their contents, are either repelled from the source droplet or attracted by the growing filaments.
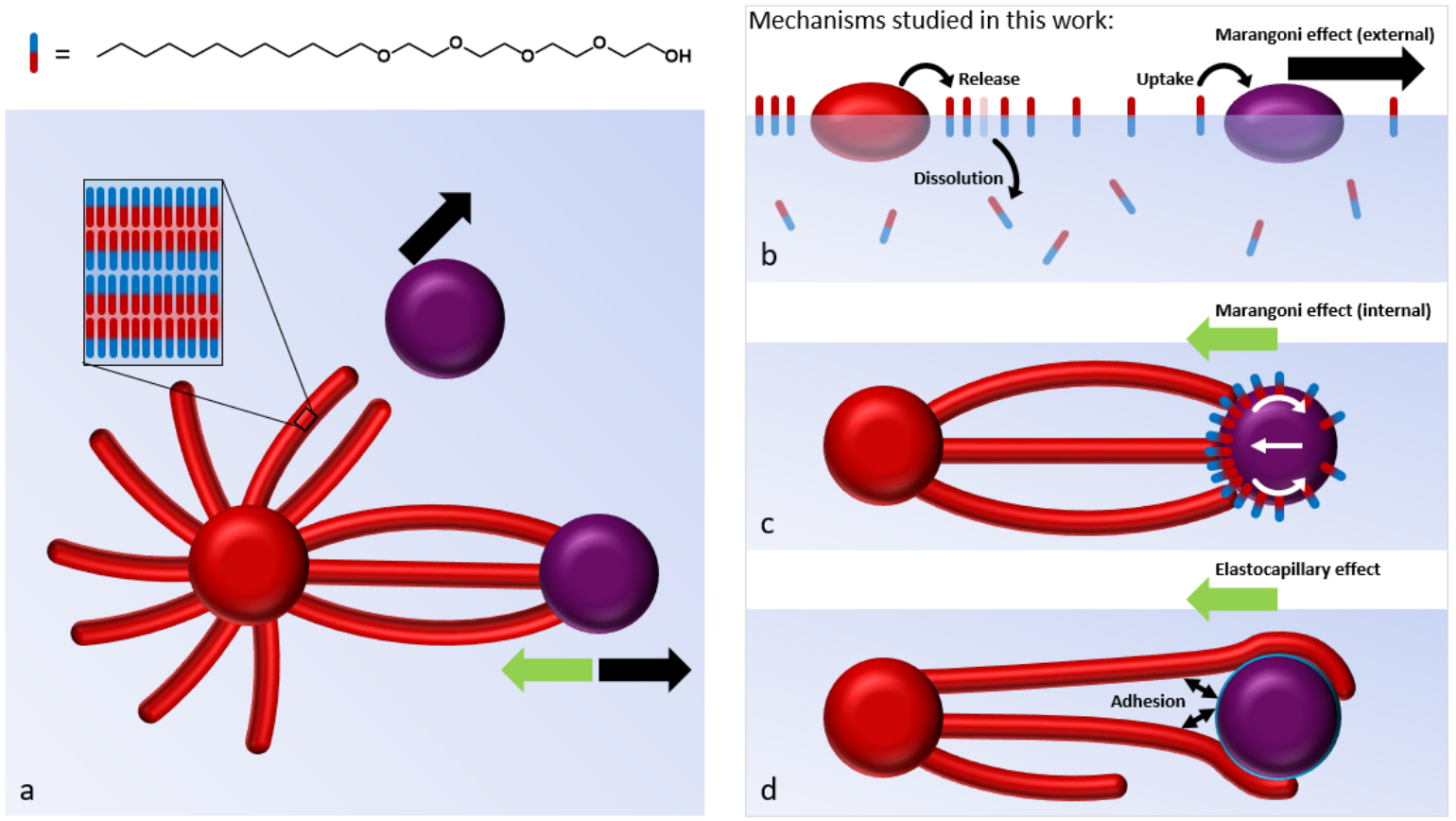

Figure 1: Self-organization mediated by a balance of repulsive and attractive forces. a) Schematic representation of filaments, composed of the amphiphile tetra(ethylene glycol) monododecyl ether $\left(\mathrm{C}_{12} \mathrm{E}_{4}\right.$, red-blue rods), that grow from the source droplet (red sphere) towards the drain droplet (purple sphere). b) $\mathrm{C}_{12} \mathrm{E}_{4}$ is released from the source to the air-water interface, and subsequently dissolved into the aqueous layer or taken up by the drain. The resulting gradient in surfactant concentration generates a flow at the air-water interface from source to drain due to the Marangoni effect, which repels the drain from the source (side view scheme). c) Absorption of a filament by the drain generates a local increase in surfactant concentration at the interface of the drain droplet. If the resulting Marangoni convection is strong enough, it propels the drain back towards the source (top view scheme). d) Alternatively, elastocapillary effects can cause filaments to wrap around a droplet. ${ }^{31}$ If the adhesive forces involved in this process are strong enough, they attract the drain towards the source (top view scheme). 


\section{Experimental Section}

Materials. Tetra(ethylene glycol) monododecyl ether was purchased from United States Pharmacopeia $(100.0 \%)$ and Santa Cruz Biotechnology Inc. (Dallas, TX) ( $\geq 98.0 \%)$. Sodium alginate, Oil red O, $4 \AA$ molecular sieves (4-8 mesh), Solvent green 3 ( $\geq 95 \%$ ) and red fluorescent sulfate-modified polystyrene beads $(0.1 \mu \mathrm{m}$, aqueous suspension) were purchased from Sigma-Aldrich, sodium chloride $(\geq 99.6 \%)$ from Fisher Chemical, oleic acid from Fluorochem $(\geq 95 \%)$ and Sigma-Aldrich $(\geq 99 \%)$, and sodium oleate $(\geq 95 \%)$ from ABCR GmbH (Karlsruhe, Germany). Red fluorescent (BODIPY605) PMMA particles with a diameter of 1-4 $\mu \mathrm{m}$ were kindly provided by dr. Evan Spruijt (Radboud University Nijmegen) and prepared according to procedures reported previously in literature. ${ }^{45,46}$ All materials were used as received.

Study of self-organization with microscopy. Filament growth was initiated following a procedure reported previously, ${ }^{41}$ by depositing $1.0 \mu \mathrm{L} \mathrm{C}_{12} \mathrm{E}_{4}$ droplets with a Gilson pipette at the interface of the medium solution in a polystyrene petri dish (lid of a Falcon $35 \mathrm{~mm}$ dish, diameter $38 \mathrm{~mm}$, height 4.5 $\mathrm{mm}$, used as received). In order to keep the droplet from moving towards the solution meniscus at the edge of the petri dish, the dish was generally filled completely with $5.5 \mathrm{~mL}$ medium solution, consisting of sodium alginate $(\mathrm{NaAlg}, 6.25 \mathrm{mg} / \mathrm{mL})$ and sodium chloride $(\mathrm{NaCl}, 17 \mathrm{mM})$ in MilliQ water, such that a convex air-water interface was formed. For experiments using molecular sieves $4.0 \mathrm{~mL}$ medium solution was used instead, and for confocal microscopy experiments, $1.75 \mathrm{~mL}$ medium solution was used to accommodate the limited working distance. Prior to the deposition of any source droplet, the surface tension of the medium solution was decreased to approx. $28 \mathrm{mN} \mathrm{m}^{-1}$ by adding a trace amount of $\mathrm{C}_{12} \mathrm{E}_{4}$ to the interface. All experiments were performed at room temperature. The formation of the lamellar phase of $\mathrm{C}_{12} \mathrm{E}_{4}$ in our experiments was verified by the opaque appearance, to the naked eye, of the source droplet after deposition on the medium solution. A $1.0 \mu \mathrm{L}$ drain droplet or $4 \AA$ molecular sieve was deposited shortly after growth of filaments had started. Further details regarding the microscopy experiments are described in the Supporting Information.

Surface tension measurements. The surface tension kinetics were investigated using a KSV Instruments LTD surface tensiometer that measures the force exerted by the air-water meniscus on a platinum Wilhelmy plate $(19.62 \mathrm{~mm} \times 10 \mathrm{~mm})$ inserted into the aqueous solution. The Wilhelmy plate was cleaned with ethanol, exposed to an open flame until red hot, and equilibrated in air. The plate was then lowered into a polystyrene petri dish (Falcon $35 \mathrm{~mm}$, height $9.5 \mathrm{~mm}$, used as received) containing $5 \mathrm{~mL}$ of $\mathrm{NaCl}(17 \mathrm{mM})$ and $\mathrm{C}_{12} \mathrm{E}_{4}(522 \mu \mathrm{M})$ in water, such that the water wetted the plate and formed a meniscus. The setup was left to equilibrate for a minimum of 12 minutes, after which $1.0 \mu \mathrm{L}$ drain solution (or a $4 \AA$ molecular sieve) was deposited near the wall of the petri dish. Except for the $4 \AA$ molecular sieve, which remained afloat in between the platinum plate and the wall, all drains moved towards the meniscus at the edge of the petri dish.

Particle image velocimetry experiments. For particle image velocimetry (PIV) analysis of the flow in the medium, red fluorescent polystyrene beads dispersed in water were included in the medium $(50 \mathrm{ppm}$ beads by weight). Samples were illuminated using a $120 \mathrm{~W}$ mercury lamp, combined with an excitation filter of 560/18 nm (Semrock Brightline) and an emission filter of 599/59 nm (Semrock Brightline). Side-view optical microscopy images and movies were acquired using a Dino-Lite AM4515ZT microscope. Bottom-view optical microscopy images and movies were acquired using an Andor iXon+ EM-CCD camera. For PIV analysis of the flow in the drain, $100 \mathrm{ppm}(\mathrm{w} / \mathrm{v})$ red fluorescent PMMA microparticles were included inside the drain, $50 \mathrm{~mW} 488$ and $561 \mathrm{~nm}$ laser beams were used simultaneously for excitation, emission was measured at 581-643 nm and movies were acquired using an Andor iXon3 EM-CCD camera. PIV analysis was performed using version 2.38 of the PIVlab plugin for MATLAB ${ }^{47}$ For PIV analysis of the flow in the drain droplets, a comparable approach was followed; further details regarding the methodology are described in the Supporting Information. 


\section{Results and Discussion}

\subsection{Marangoni flow in the aqueous medium surrounding the drain droplet.}

First, we investigated how the Marangoni flow directs the movement of the drain droplet and, vice versa, how the uptake of surfactant molecules by said drain affects the Marangoni flow. To this end, we characterized the flow profile in the aqueous medium via particle image velocimetry. In our setup, the system was illuminated by a laser from the bottom, and fluorescence was emitted by polystyrene microparticles dispersed in the solution. This allowed us to acquire side view profiles of the flow, as schematically shown in Figure 2a, which were analyzed using the PIVlab plugin for MATLAB. ${ }^{47}$ Immediately after the deposition of the $\mathrm{C}_{12} \mathrm{E}_{4}$ source droplet on top of a $\mathrm{NaAlg} / \mathrm{NaCl}$ medium solution, a Marangoni flow emerges (Figure 2b). At the air-water interface, the flow moves to the right, away from the source droplet, with a velocity of approx. $10 \mu \mathrm{m} \mathrm{s}^{-1}$. A few millimeters under the water surface, a backflow emerges in the opposite direction, towards the source droplet.

Upon deposition of a drain droplet that consists of $10 \mathrm{wt} \%$ sodium oleate in oleic acid $(10 \%$ $\mathrm{NaOleate} / \mathrm{OA}$ ), the Marangoni flow velocity increases up to approx. $20 \mu \mathrm{m} \mathrm{s}^{-1}$ (Figure 2c), indicating that the drain depletes surfactant from the air-water interface. Remarkably, even though the flow is directed from the source to the drain, the drain droplet moves against this flow towards the source at a rate of approx. $4 \mu \mathrm{m} \mathrm{s}^{-1}$ (Figure 2d-e, Movie S1). Side view images of the flow profile (Figure 2f) indicate that the motion of the drain droplet cannot be attributed to the backflow, as the floating droplet is not submerged deeply enough to be influenced by this flow. At the rear of the drain droplet, the interfacial flow is directed such that it would push back the drain to the source. However, a bottomview PIV analysis of a drain droplet reveals that the net flow of the fluid surrounding the drain droplet is still directed such that it would push the drain away from the source droplet (Figure 2g,h, Movie S2).

Together, these results show that the Marangoni flow from source to drain can be amplified by the uptake of surfactant molecules at the drain. Moreover, an additional force is anticipated to act on the drain droplet, driving its motion opposite to the Marangoni flow, towards the source. 

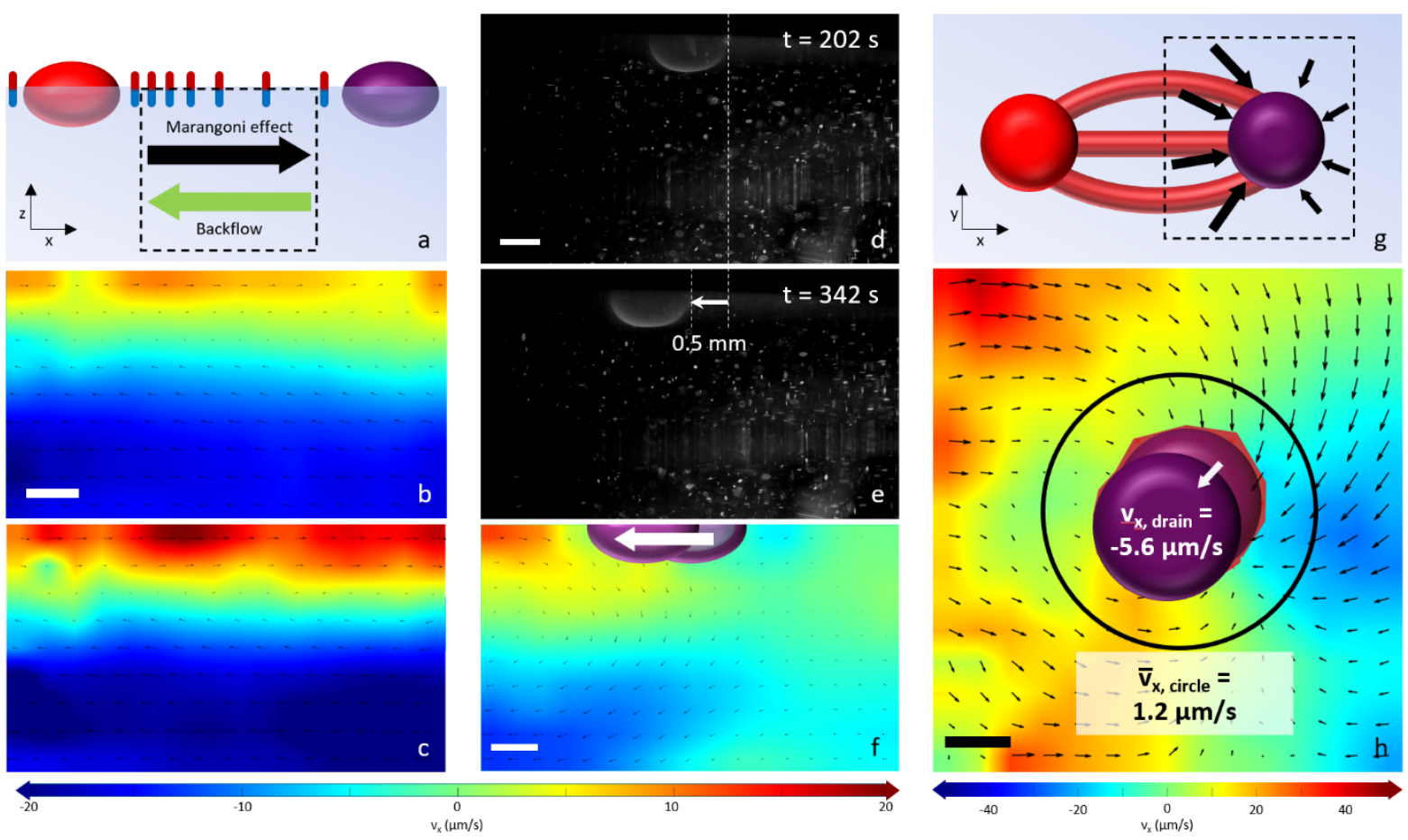

Figure 2. Visualization of the Marangoni effect and backflow in the aqueous solution surrounding the drain droplet. a) Schematic representation of the self-organizing source-drain system (side view). The dashed box represents the section of the system that is studied in the particle image velocimetry (PIV) analysis. b, c) PIV analysis of the vertical flow velocity profile (i.e. $v_{\mathrm{x}} v$ s. $\mathrm{x}$ and $z$ ) in the aqueous solution in between the source and drain, before (b) and after (c) deposition of a $10 \mathrm{wt} \%$ sodium oleate in oleic acid ( $10 \% \mathrm{NaOleate} / \mathrm{OA})$ drain droplet. Upon deposition of the drain droplet, the flow velocity increases. d, e) Fluorescence microscopy images (side view) representing the first and last frame used for ensemble analysis in (f). The fluorescent tracer particles present in the medium show up as bright dots. Over time, the drain moves to the left, where the source is located. f) PIV ensemble analysis of the flow velocity profile in the vicinity of the drain droplet. g) Schematic representation of the selforganizing source-drain system (bottom view). The dashed box represents the section of the system that is studied in the PIV analysis. h) PIV ensemble analysis of the horizontal flow velocity profile (i.e. $v_{\mathrm{x}}$ $v s . \mathrm{x}$ and $\mathrm{y}$ ), in the vicinity of a $10 \%$ NaOleate/OA drain droplet. The average magnitude of $v_{\mathrm{x}}$, taken over the black circle around the drain, equals $1.2 \mu \mathrm{m} / \mathrm{s}$; the velocity of the drain in the x-direction equals $-5.6 \mu \mathrm{m} / \mathrm{s}$. All scale bars represent $500 \mu \mathrm{m}$.

\subsection{Interaction between the drain droplet and filaments}

The motion of the drain droplet opposite to the direction of the flow in the aqueous solution prompted us to investigate how the filaments, which move along the Marangoni flow, interact with the drain upon arrival. Following the mechanisms discussed in the introduction, the motion of the drain back to the source can be driven by both propulsion through 1) internal Marangoni flow (Figure 1c) and 2) elastocapillary effects (Figure 1d). The first mechanism implies absorption of filaments by the drain, such that $\mathrm{C}_{12} \mathrm{E}_{4}$ amphiphiles are delivered which occupy the water-oil interface and act as a surfactant. The second mechanism implies that the filaments remain intact and adhere to the drain droplet. To characterize the absorption or adhesion of filaments at the drain, experiments were conducted with $\mathrm{C}_{12} \mathrm{E}_{4}$ source droplets containing the dye Oil Red O. Within 90 seconds after deposition of the source, a drain droplet was deposited at the air-water interface. Interactions were studied for three different types of drain: 1) pure oleic acid (OA); 2) $10 \%$ NaOleate/OA; and 3) oleic acid with $10 \% \mathrm{v} / \mathrm{v} \mathrm{C}_{12} \mathrm{E}_{4}$ $\left(10 \% \mathrm{C}_{12} \mathrm{E}_{4} / \mathrm{OA}\right)$. 

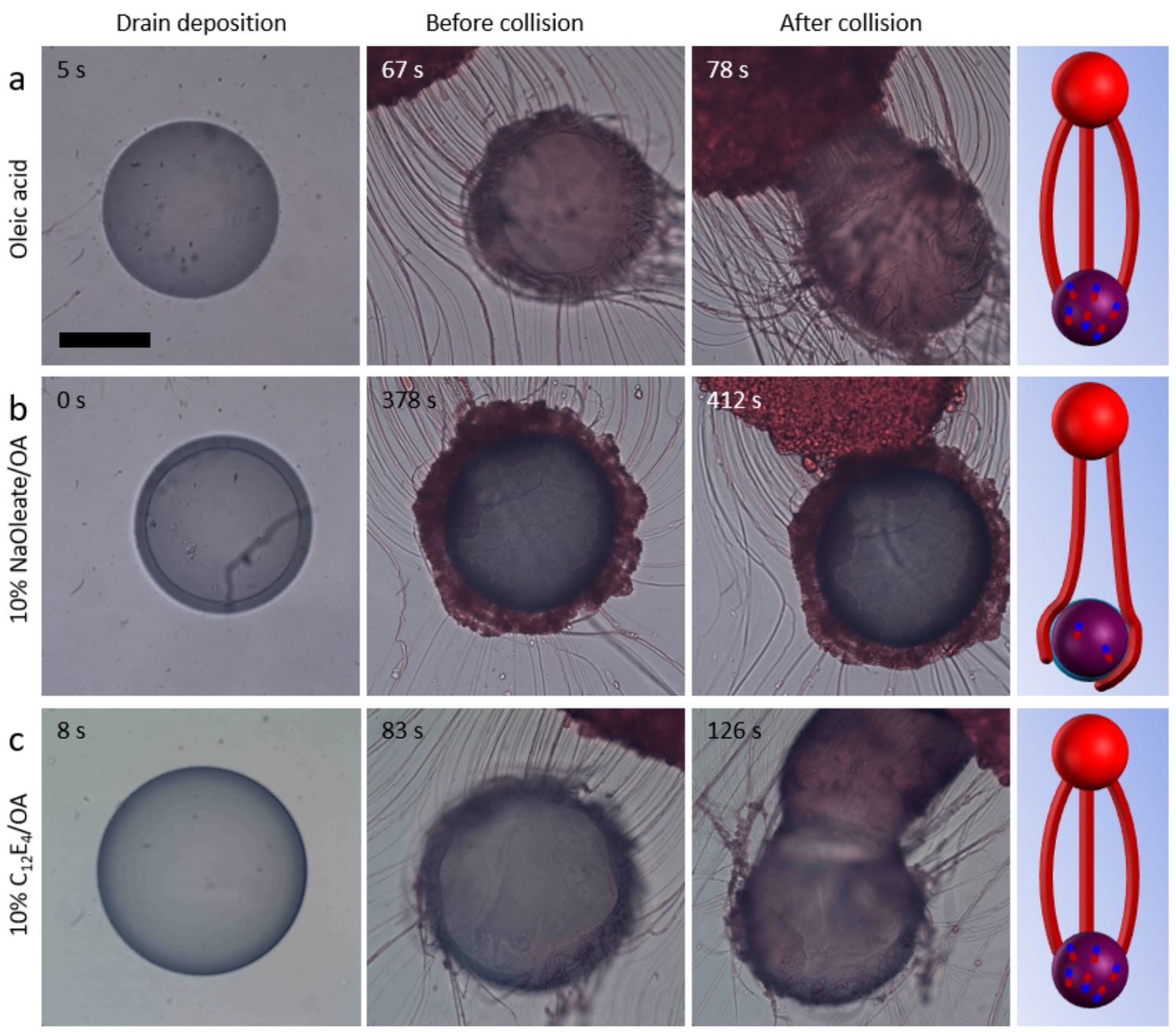

Figure 3. Interaction between the filaments and the drain droplet: absorption vs. adhesion. Optical microscopy images of three different drain types: a) pure oleic acid (OA); b) $10 \% \mathrm{NaOleate} / \mathrm{OA}$ and c) $10 \% \mathrm{v} / \mathrm{v} \mathrm{C}_{12} \mathrm{E}_{4}$ in $\mathrm{OA}\left(10 \% \mathrm{C}_{12} \mathrm{E}_{4} / \mathrm{OA}\right)$ drain droplets upon deposition (left column) as well as right before (middle column) and after (right column) collision with a source droplet dyed with Oil Red O. Filaments accumulate around the $10 \%$ NaOleate/OA drain, while they get absorbed by the other drain types. The scale bar represents $500 \mu \mathrm{m}$, and all drain droplets were deposited at $t=0 \mathrm{~s}$.

As shown in Figure 3a and Movie S3 (left panel), the OA drain rapidly attracts the filaments, and upon contact acquires a red color, which is indicative for their absorption. After collision with the source droplet, the deep red coloring of the drain suggests a further absorption of material from the source. Intriguingly, for a $10 \% \mathrm{NaOleate} / \mathrm{OA}$ drain, all filaments accumulate at the edge of the droplet (Figure 3b, middle panel of Movie S3). The red color does not penetrate into the opaque drain droplet, even when the drain and source collide, indicating that no material of the filaments is absorbed by the drain. This can be rationalized by the formation of a liquid crystalline film at the interface of the droplet with the surrounding aqueous phase. $\mathrm{Fd} 3 \mathrm{~m}$ and $\mathrm{L}_{2}$ liquid crystalline phases have been reported in literature when diluting $10 \% \mathrm{NaOleate} / \mathrm{OA}$ with an aqueous $\mathrm{NaCl}$ solution. ${ }^{48}$ Indeed, when a $10 \% \mathrm{NaOleate} / \mathrm{OA}$ solution was carefully applied on top of a $\mathrm{NaAlg} / \mathrm{NaCl}$ solution, the interface was rapidly covered by a thin film. In the absence of NaOleate, no film formation was observed at the interface of OA and the $\mathrm{NaAlg} / \mathrm{NaCl}$ solution. Surface tension measurements using the Wilhelmy plate method and PIV analysis reveal that the film obstructs the uptake of $\mathrm{C}_{12} \mathrm{E}_{4}$ from the air-water interface by the drain 
droplet. Compared to $10 \%$ NaOleate/OA drains, pure OA drains display an enhanced uptake of $\mathrm{C}_{12} \mathrm{E}_{4}$ molecules from the air-water interface, and thus an increased Marangoni flow velocity (Figure S1 and S2, Movie S7).

To investigate the relation between film formation and accumulation of filaments at the drain boundary, $\mathrm{C}_{12} \mathrm{E}_{4} / \mathrm{OA}$ drain droplets were studied. This type of drain does not form a film when in contact with the $\mathrm{NaAlg} / \mathrm{NaCl}$ solution, yet surface tension measurements (Figure $\mathrm{S2}$ ) indicate that $\mathrm{C}_{12} \mathrm{E}_{4}$ uptake from the air-water interface is decreased in comparison to a pure OA drain. As shown in Figure 3c, as well as the right panel of Movie S3, a $10 \% \mathrm{C}_{12} \mathrm{E}_{4} / \mathrm{OA}$ drain absorbs filaments loaded with Oil red $\mathrm{O}$, demonstrated by the coloring of the droplet interior. However, the absorption of filaments is incomplete and some of the filaments accumulate in clusters at the edge of the droplet. Together, the results indicate that inclusion of surfactants, i.e. NaOleate or $\mathrm{C}_{12} \mathrm{E}_{4}$, reduces the activity of the drain in terms of interfacial $\mathrm{C}_{12} \mathrm{E}_{4}$ uptake as well as filament absorption.

\subsection{Marangoni flow inside the drain droplet.}

Internal Marangoni flow as a mechanism to propel the drain towards the source relies on delivery of $\mathrm{C}_{12} \mathrm{E}_{4}$ at the oil-water interface by the filaments. Therefore, we assessed whether the differences in filament absorption observed for the three drain types result in differences in the internal Marangoni flow. To quantify the internal flow in the drain upon arrival of $\mathrm{C}_{12} \mathrm{E}_{4}$ filaments, PIV analysis was performed for each drain type. For pure OA and 10\% NaOleate/OA drains, fluorescent PMMA particles were dispersed in the drain droplet and confocal microscopy was used to visualize their movement. In $10 \% \mathrm{C}_{12} \mathrm{E}_{4} / \mathrm{OA}$ drains, the presence of phase-separated microdroplets allowed us to observe convection patterns using optical microscopy.

For OA drains (Figure 4a,d, Movie S4a), internal convection patterns emerge in PIV, and the occurrences of these transient flow patterns coincide with the irregular motion of the droplet. The flow can be attributed to filaments that arrive at the droplet, get absorbed by the drain, and locally generate an excess of $\mathrm{C}_{12} \mathrm{E}_{4}$ at the oil-water interface at the site of the absorption event. We note that the internal flow reaches velocities up to $1.3 \mathrm{~mm} \mathrm{~s}^{-1}$, and thereby dominates the motion of the droplet. In contrast, for the $10 \%$ NaOleate/OA drain droplet (Figure 4b,e, Movie S4b), the internal flow in the drain is multiple orders of magnitude lower than the flow velocities measured in the surrounding medium (Figure 2h), and hence not sufficient to drive the drain towards the source. We attribute this reduced flow velocity to the liquid crystalline film at the interface of the drain, which prevents absorption of filaments, such that no significant surface tension gradient is generated at the oil-water interface. For the $10 \% \mathrm{C}_{12} \mathrm{E}_{4} / \mathrm{OA}$ drain (Figure 4c,f, Movie $\mathbf{S 4 c}$ ), the intensity of the internal convection patterns was similar to those found for pure $\mathrm{OA}$. While inclusion of $\mathrm{C}_{12} \mathrm{E}_{4}$ in the drain hinders the uptake of filaments, as demonstrated in Figure 3c, the Marangoni-driven convection generated upon absorption of a filament is undiminished.

Together, these results show that an internal Marangoni flow is generated when the drain droplet absorbs filaments, and that this Marangoni effect can be manipulated by the inclusion of NaOleate in the drain. However, the irregularity of convection events, both in terms of frequency and direction, as well as the fact that the drain droplets display constant motion towards the source even when little or no convection is observed, indicate that internal Marangoni convection is not the only driving force involved in the attraction of drain droplets towards the source. 


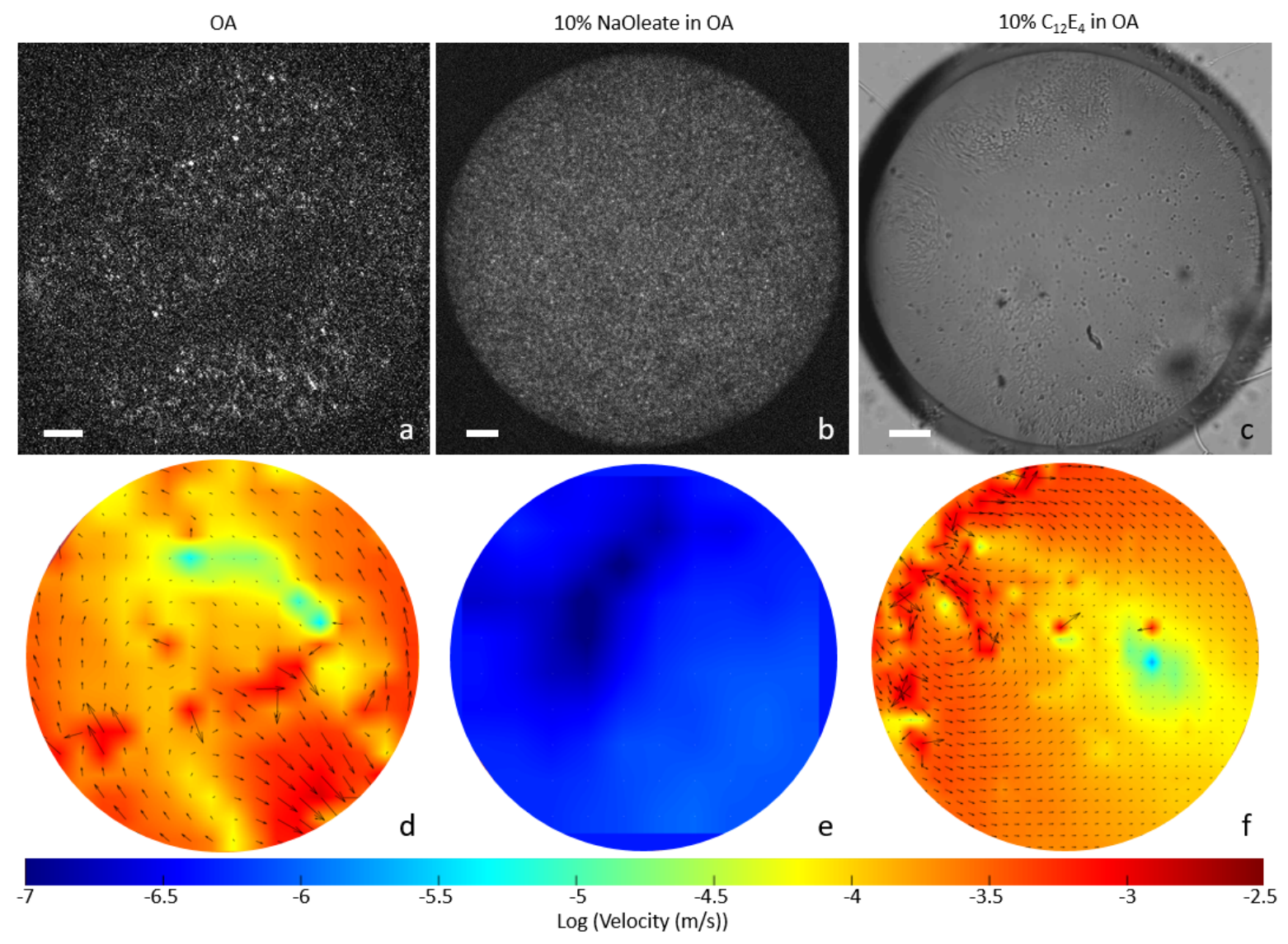

Figure 4. Visualization of the Marangoni effect within the drain droplet. Microscopy images of a) a pure $\mathrm{OA}$ drain; b) a $10 \% \mathrm{NaOleate} / \mathrm{OA}$ drain and c) a $10 \% \mathrm{C}_{12} \mathrm{E}_{4} / \mathrm{OA}$ drain. The $\mathrm{OA}$ and NaOleate/OA drains were loaded with fluorescent PMMA particles, visualized via confocal microscopy. The images of the $\mathrm{C}_{12} \mathrm{E}_{4} / \mathrm{OA}$ drain were acquired using optical microscopy. d-f) PIV analysis of the velocity profiles corresponding to the drain droplets in the top row. No significant flow is detected for the $10 \%$ NaOleate/OA drain, while clear convection patterns are observed in the $\mathrm{OA}$ and $10 \% \mathrm{C}_{12} \mathrm{E}_{4} / \mathrm{OA}$ drains. The scale bars represent $100 \mu \mathrm{m}$.

\subsection{Attraction of the drain by elastocapillary effects.}

Next, we study the role of elastocapillary effects in source-drain attraction. Recently, Prasath et al. ${ }^{31}$ demonstrated that a thin floating polydimethylsiloxane filament (diameter approx. $100 \mu \mathrm{m}$ ) wraps around a floating oil droplet, as capillary attraction provides the energy required to bend the filament. In our source-drain system, the tension on the filaments resulting from these forces potentially pulls the drain towards the source, as the filaments wrap themselves around the drain without being absorbed (Figure 1d). To test this hypothesis, we eliminated the internal Marangoni effect in the drain by using solid spherical zeolite-based $4 \AA$ molecular sieves (MolSieve) instead of oil droplets. A MolSieve is wetted by water, while also retaining the ability to float. Whereas the pore size of the MolSieve is too small to absorb $\mathrm{C}_{12} \mathrm{E}_{4}$ from the air-water interface (Figure S2), the medium flows towards the drain, as water is absorbed (Figure S3 and Movie S8). When a MolSieve is deposited on an air-water interface at which a source droplet is already present (Figure 5a, Movie S5), filaments are attracted towards it due to this flow. Initially, the drain moves away from the source, driven by the outward Marangoni flow in the aqueous solution. Subsequently, at approx. 1000 seconds, filaments connect to the MolSieve, which is then kept in its position, despite the fact that filaments are still growing from the source, as 
shown in Figure 5c. After approx. 80 minutes, the drain stops collecting filaments, although filaments which were already connected remain attached, and the drain moves away from the source as the filaments keep growing.

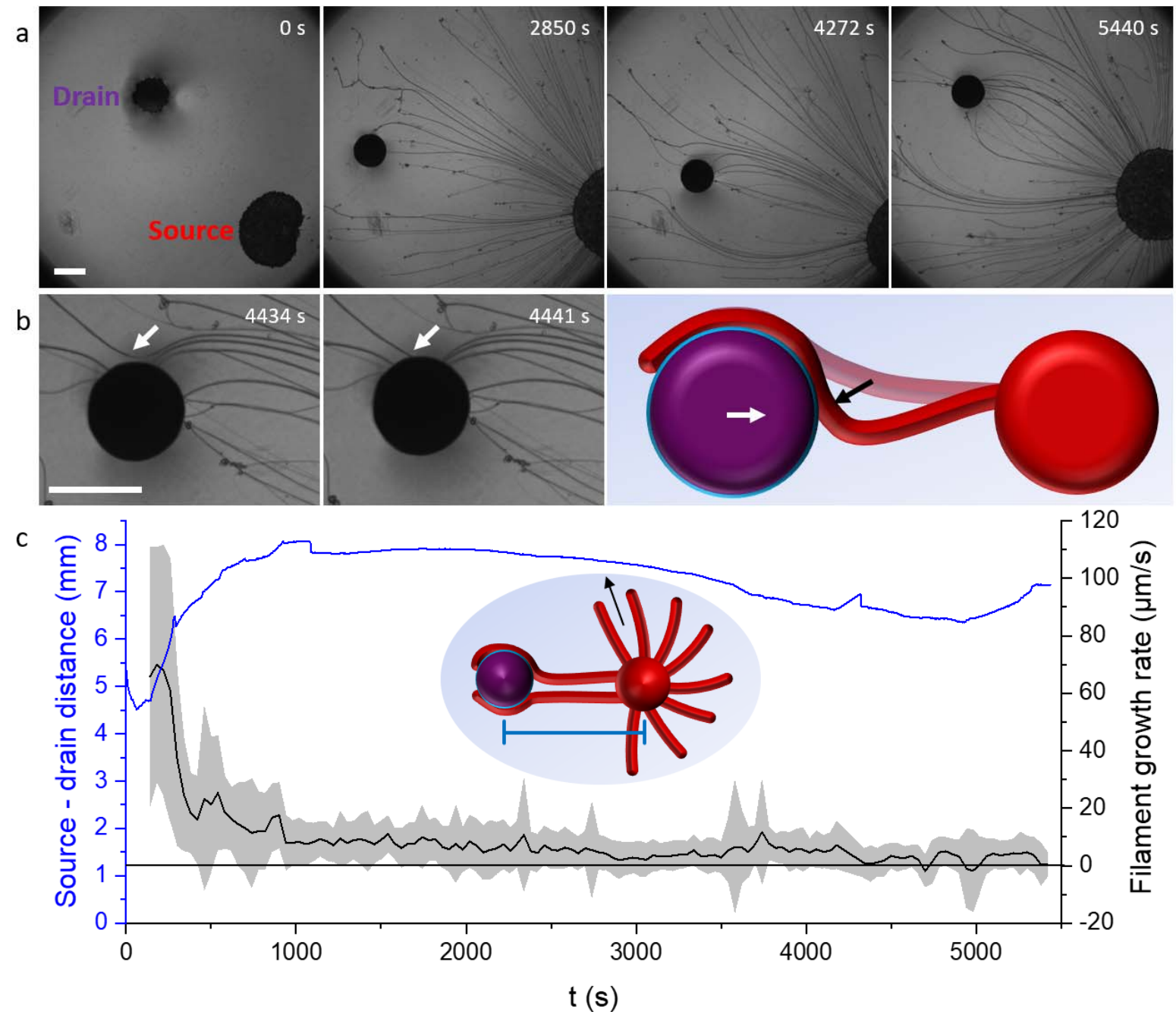

Figure 5. Attraction of a solid molecular sieve drain by elastocapillary effects acting on the filaments. a) Optical microscopy images acquired on the self-organization of a $\mathrm{C}_{12} \mathrm{E}_{4}$ source and a $4 \AA$ molecular sieve that was applied as drain at the air-water interface. b) Close-up images of the $4 \AA$ molecular sieve, showing how a filament (indicated with arrow) approaches towards and attaches to the drain. The scheme showcases the proposed mechanism for source-drain attraction based on elastocapillary effects. c) Center-to-center distance between the source and the drain, as well as the average growth rate of the filaments vs. time. Initially, the drain is repelled from the source. When filaments attach to the drain, the distance between the source and drain remains relatively constant, even though the filaments are still growing from the source - as schematically shown in the inset. Filament growth rate was approximated by tracking the movement of defects present on the filaments relative to the source (see Supplementary Information for details). The scale bars represent $1 \mathrm{~mm}$.

The study of the interaction between filaments and the MolSieve drain is impaired by the opaque nature of the molecular sieve particle. Therefore, we further unravel the attraction of the filaments via elastocapillary effects by using a $20 \mathrm{v} / \mathrm{v} \% \mathrm{C}_{12} \mathrm{E}_{4}$ in OA drain droplet. The increased content of $\mathrm{C}_{12} \mathrm{E}_{4}$ 
reduces the rate at which the filaments are absorbed in comparison to a $10 \% \mathrm{C}_{12} \mathrm{E}_{4} / \mathrm{OA}$ drain (Figure S2). As shown in Figure 6 and Movie S6, many filaments wrap around the drain, rather than being absorbed - this can also be observed from the sideways approach of filaments towards the MolSieve in Figure 5b. Subsequently, as these filaments accumulate at the drain, they also stick to each other and form a ring of filaments wrapped around the drain. When filaments pull from multiple directions at once, the filament ring can be partially separated from the drain while remaining intact, indicating that there may be limited contact between filaments and the droplet, and that filaments attach directly to the clustered filaments in the ring (Figure S4, Movie S9). Together, these observations support a mechanism in which drain droplets are attracted to the source droplet through elastocapillary forces, mediated by a cluster of filaments adhering to the drain.
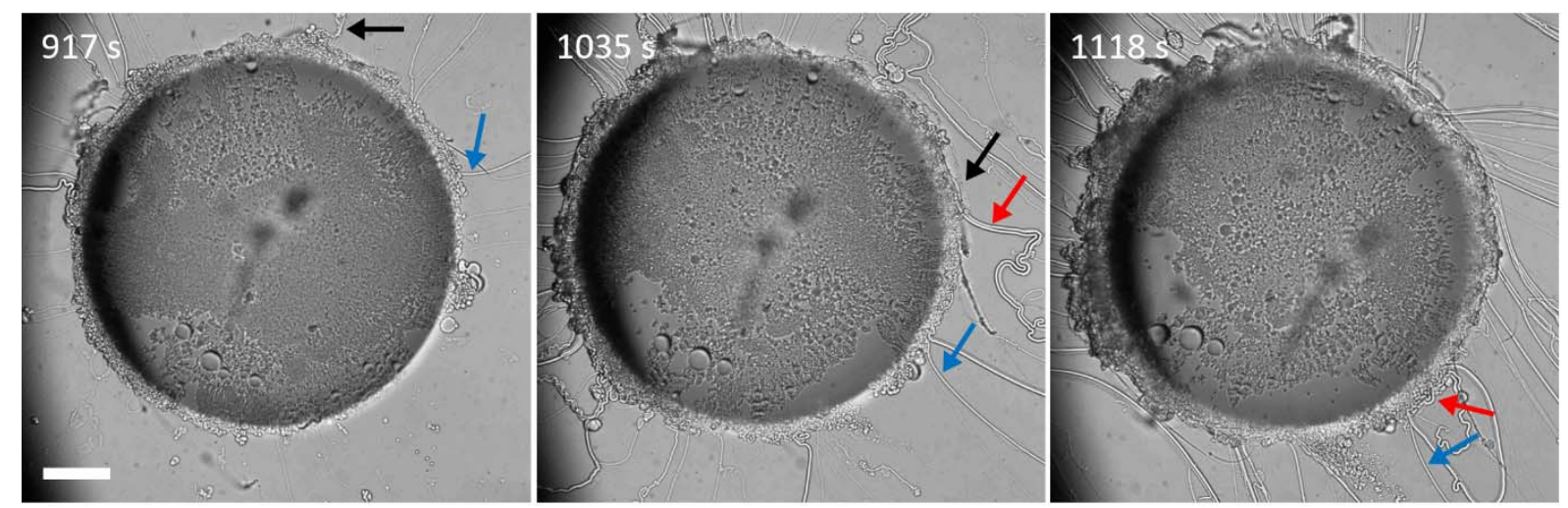

Figure 6. Elastocapillary adhesion of filaments to drain droplets. Optical microscopy images of filaments adhering to a $20 \% \mathrm{C}_{12} \mathrm{E}_{4} / \mathrm{OA}$ drain droplet. Inclusion of $\mathrm{C}_{12} \mathrm{E}_{4}$ reduces the absorption rate, such that $\mathrm{C}_{12} \mathrm{E}_{4}$-based filaments remain intact upon contact with the drain. Three distinct filaments are indicated with black, blue and red arrows, respectively, as they wrap themselves around the drain and cluster together. The scale bar represents $200 \mu \mathrm{m}$.

\subsection{Selective attraction of drain droplets by the filaments.}

Finally, we attempt to demonstrate the unique behavior enabled through the connectivity of the filaments by establishing a source droplet that selectively attracts floating drain droplets, based on their chemical composition. The release of $\mathrm{C}_{12} \mathrm{E}_{4}$ from the source droplet generates an outward Marangoni flow, resulting in the repulsion of said drain (Figure 7a). Simultaneously, the tethering of filaments to the drain can, either via capillary effects or an internal Marangoni flow, keep the drain in position (Figure 7b) or even withdraw the drain towards the source (Figure 7c). As the flow that carries filaments to the drain depends on the rate at which the drain removes $\mathrm{C}_{12} \mathrm{E}_{4}$ from the air-water interface, we used three different drain droplets $\left(10 \% \mathrm{C}_{12} \mathrm{E}_{4} / \mathrm{OA}, 20 \% \mathrm{C}_{12} \mathrm{E}_{4} / \mathrm{OA}\right.$ and $\left.30 \% \mathrm{C}_{12} \mathrm{E}_{4} / \mathrm{OA}\right)$ in an attempt to tune the strength of attraction. Surface tension measurements show how the inclusion of $\mathrm{C}_{12} \mathrm{E}_{4}$ decreases the rate at which an OA-based drain depletes $\mathrm{C}_{12} \mathrm{E}_{4}$ from the air-water interface (Figure 7d). Indeed, when a $\mathrm{C}_{12} \mathrm{E}_{4}$-based source droplet and four drain droplets are simultaneously deposited at the air-water interface (Figure 7e-g, Figure S5), a larger $\mathrm{C}_{12} \mathrm{E}_{4}$ content in the drain results in a smaller amount of filaments being attracted toward the drain. The $10 \% \mathrm{C}_{12} \mathrm{E}_{4} / \mathrm{OA}$ drain is tethered to a large number of filaments, which draw the droplet into a position close to, or even in contact with, the source. The $20 \% \mathrm{C}_{12} \mathrm{E}_{4} / \mathrm{OA}$ drain remains further away from the source, even though it is tethered to a number of filaments, whereas the $30 \% \mathrm{C}_{12} \mathrm{E}_{4} / \mathrm{OA}$ drains are not connected to the source at all, and are repelled. 


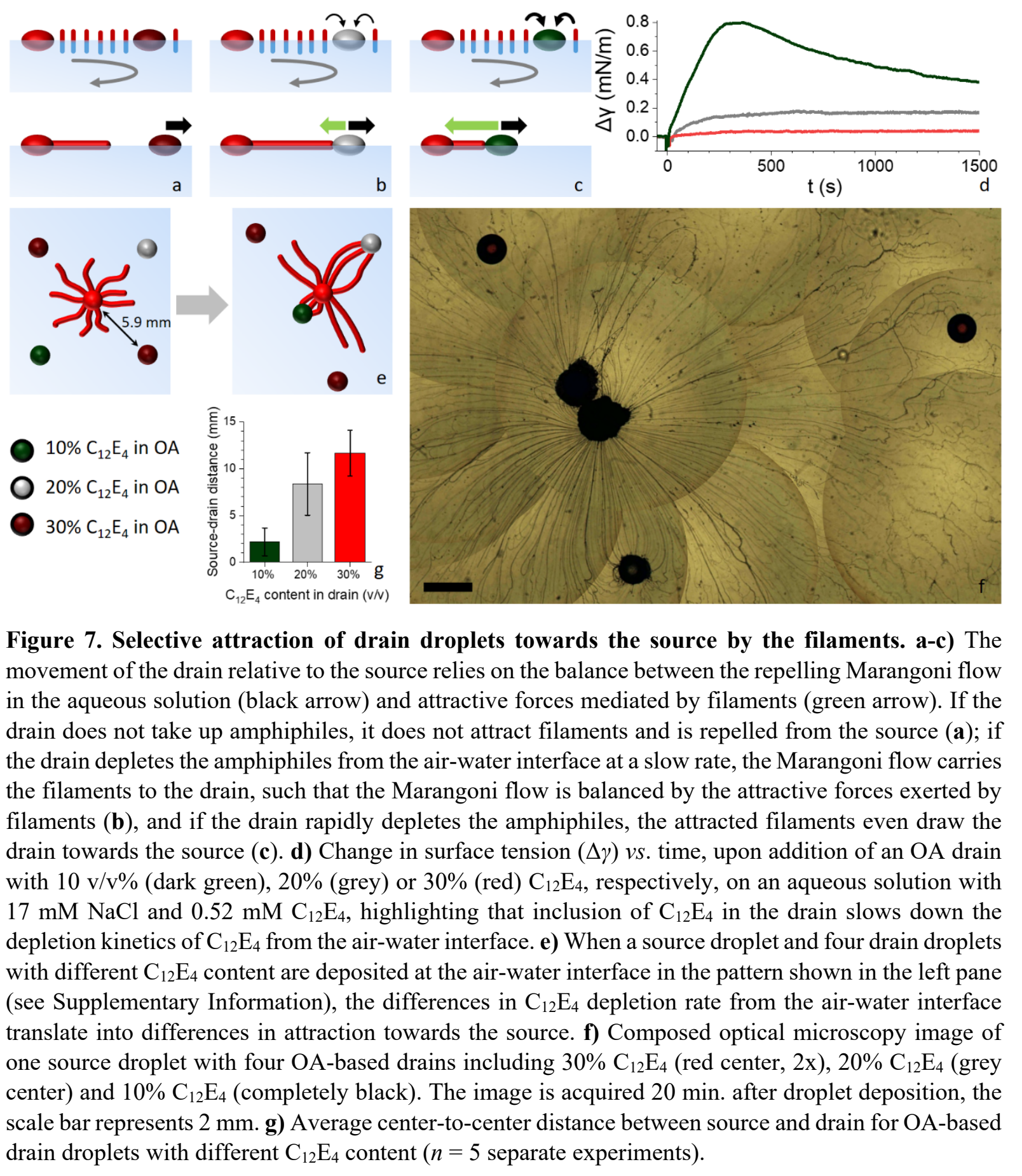

\section{Conclusions}

We unraveled how Marangoni and elastocapillary effects direct the self-organization of floating droplets and amphiphile filaments at air-water interfaces. First, the release of $\mathrm{C}_{12} \mathrm{E}_{4}$ amphiphiles from an amphiphile source droplet drives the Marangoni flow in the aqueous solution, which was successfully visualized through PIV analysis. Then, if a drain is deposited onto the solution as well, the Marangoni flow initially repels the droplet from the source. At the same time, the uptake of individual amphiphiles from the air-water interface by the drain directs the Marangoni flow towards the drain, thereby attracting the amphiphile filaments that grow from the source. Subsequently, the filaments that 
contact the drain can exert an attractive force via two distinct mechanisms: 1) internal Marangoni flow in the drain fluid, and 2) elastocapillary effects.

Upon absorption of the filaments by an OA-based drain, the release of $\mathrm{C}_{12} \mathrm{E}_{4}$ to the oil-water interface drives a strong internal Marangoni convection - with much higher velocity than the flow in the surrounding aqueous phase - in the liquid drain droplet. As a consequence, the drain is propelled towards the absorption site. Although the forces involved in this process are too large and their occurrence too irregular to allow for stable self-organization, their impact can be diminished through inclusion of NaOleate or $\mathrm{C}_{12} \mathrm{E}_{4}$ in the drain. NaOleate forms a liquid crystalline film which blocks absorption of filaments, thus preventing internal flow altogether; $\mathrm{C}_{12} \mathrm{E}_{4}$, on the other hand, slows down convection by hindering filament absorption.

Next, attraction can be directed through elastocapillary effects, when the filaments are not absorbed by the drain. For a molecular sieve, we demonstrated how the adhesion of filaments contributes to their positioning and that internal Marangoni flow is not required for self-organization. Finally, a closer look at a $20 \% \mathrm{C}_{12} \mathrm{E}_{4} / \mathrm{OA}$ drain reveals that some filaments fold themselves around the drain - rather than being absorbed, becoming part of a growing circular cluster of filaments around the drain. Together, these results show that elastocapillary effects are at play, and that tensile forces can be transferred, through filaments, to the filament cluster and the drain to which it adheres.

We note that the emergence of gradients offers a general design principle to establish spatial differentiation in self-organization processes. The release of surface active compounds from floating objects has been exploited to generate Marangoni flows that drive their mutual repulsion, and thereby the self-organization. ${ }^{49}$ However, whereas such Marangoni flow would result in repulsion of all floating objects, these amphiphile filaments - which connect only to some of the floating droplets - can exert selective forces by which the floating droplets are attracted. Together, these findings were employed to create a self-organizing system in which a source droplet differentiates between drain droplets based on their composition, by selectively forming connections between elements which, through said connections, establish self-organization. Understanding the physicochemical phenomena at play in interfacial source-drain systems paves the way for the construction of out-of-equilibrium selforganizing networks with dynamic connectivity, for example by including dissipative source and/or drain elements, larger numbers of droplets, as well as other mechanisms that lead to pattern formation. In doing so, we start to bridge the gap between synthetic and biological systems, ultimately distilling their complex functionalities from simple building blocks.

\section{Supporting information}

Additional experimental details, Supplementary Figures and Supplementary movies corresponding to the microscopy images.

\section{Acknowledgements}

We thank the Dutch Research Council (NWO, START-UP grant no. 740.018.003) as well as the Dutch Ministry of Education, Culture and Science (Gravitation program 024.001.035) for financial support. We also thank Anne-Déborah Nguindjel, Pieter de Visser and prof. dr. Wilhelm Huck for stimulating discussions, and dr. Evan Spruijt for kindly providing fluorescent PMMA particles used in the PIV analysis. 


\section{Author contributions}

M.W. performed the experiments and analysis. P.A.K. conceived and supervised the project. M.W. and P.A.K. wrote the paper.

\section{Notes}

The authors declare no competing financial interest.

\section{References}

(1) Vignaud, T.; Blanchoin, L.; Théry, M. Directed Cytoskeleton Self-Organization. Trends Cell Biol. 2012, 22 (12), 671-682. https://doi.org/10.1016/j.tcb.2012.08.012.

(2) Misteli, T. The Concept of Self-Organization in Cellular Architecture. J. Cell Biol. 2001, 155 (2), 181185. https://doi.org/10.1083/jcb.200108110.

(3) Tero, A.; Takagi, S.; Saigusa, T.; Ito, K.; Bebber, D. P.; Fricker, M. D.; Yumiki, K.; Kobayashi, R.; Nakagaki, T. Rules for Biologically Inspired Adaptive Network Design. Science 2010, 327 (1), 439442.

(4) Kelso, J. A. S. Dynamic Patterns : The Self-Organization of Brain and Behavior; MIT Press: Cambridge, 1995.

(5) Santos-Moreno, J.; Schaerli, Y. Using Synthetic Biology to Engineer Spatial Patterns. Adv. Biosyst. 2019, 3 (4), 1-15. https://doi.org/10.1002/adbi.201800280.

(6) Giuseppone, N. Towards Self-Constructing Materials: A Chemistry Approach. Acc. Chem. Res. 2012, $45(12), 2178-2188$.

(7) Grzybowski, B. A.; Fitzner, K.; Paczesny, J.; Granick, S. From Dynamic Self-Assembly to Networked Chemical Systems. Chem. Soc. Rev. 2017, 46 (18), 5647-5678. https://doi.org/10.1039/c7cs00089h.

(8) Kaspar, C.; Ravoo, B. J.; van der Wiel, W. G.; Wegner, S. V.; Pernice, W. H. P. The Rise of Intelligent Matter. Nature 2021, 594 (7863), 345-355. https://doi.org/10.1038/s41586-021-03453-y.

(9) Mai, A. Q.; Bánsági, T.; Taylor, A. F.; Pojman, J. A. Reaction-Diffusion Hydrogels from Urease Enzyme Particles for Patterned Coatings. Commun. Chem. 2021, 4 (1), 1-7. https://doi.org/10.1038/s42004-021-00538-7.

(10) Vanag, V. K.; Epstein, I. R. Segmented Spiral Waves in a Reaction-Diffusion System. Proc. Natl. Acad. Sci. 2003, 100 (25), 14635-14638. https://doi.org/10.1073/pnas.2534816100.

(11) Zhabotinsky, A. M. Periodical Oxidation of Malonic Acid in Solution (a Study of the Belousov Reaction Kinetics). Biofizika 1964, 9, 306-311.

(12) Horváth, J.; Szalai, I.; De Kepper, P. Designing Stationary Reaction-Diffusion Patterns in PH SelfActivated Systems. Acc. Chem. Res. 2018, 51 (12), 3183-3190.

https://doi.org/10.1021/acs.accounts.8b00441.

(13) Zhang, Y.; Tsitkov, S.; Hess, H. Complex Dynamics in a Two-Enzyme Reaction Network with Substrate Competition. Nat. Catal. 2018, 1 (4), 276-281. https://doi.org/10.1038/s41929-018-0053-1.

(14) Hendrikse, H. C.; van der Weijden, A.; Ronda-Lloret, M.; Yang, T.; Bliem, R.; Shiju, N. R.; van Hecke, M.; Li, L.; Noorduin, W. L. Shape-Preserving Chemical Conversion of Architected Nanocomposites. Adv. Mater. 2020, 32 (52), 1-7. https://doi.org/10.1002/adma.202003999.

(15) Soejima, T.; Amako, Y.; Ito, S.; Kimizuka, N. Light-Reducible Dissipative Nanostructures Formed at the Solid-Liquid Interface. Langmuir 2014, 30 (47), 14219-14225. https://doi.org/10.1021/la5036568.

(16) Cheng, X.; Ferrell, J. E. Spontaneous Emergence of Cell-like Organization in Xenopus Egg Extracts. Science 2019, 366 (6465), 631-637. https://doi.org/10.1126/science.aav7793.

(17) Thutupalli, S.; Seemann, R.; Herminghaus, S. Swarming Behavior of Simple Model Squirmers. New J. Phys. 2011, 13, 073021. https://doi.org/10.1088/1367-2630/13/7/073021.

(18) Čejková, J.; Schwarzenberger, K.; Eckert, K.; Tanaka, S. Dancing Performance of Organic Droplets in Aqueous Surfactant Solutions. Colloids Surfaces A Physicochem. Eng. Asp. 2019, 566 (January), 141147. https://doi.org/10.1016/j.colsurfa.2019.01.027.

(19) Kichatov, B.; Korshunov, A.; Sudakov, V.; Gubernov, V.; Yakovenko, I.; Kiverin, A. Crystallization of 
Active Emulsion. Langmuir 2021, 37 (18), 5691-5698. https://doi.org/10.1021/acs.langmuir.1c00630.

(20) Meredith, C. H.; Moerman, P. G.; Groenewold, J.; Chiu, Y. J.; Kegel, W. K.; van Blaaderen, A.; Zarzar, L. D. Predator-Prey Interactions between Droplets Driven by Non-Reciprocal Oil Exchange. Nat. Chem. 2020, 12 (12), 1136-1142. https://doi.org/10.1038/s41557-020-00575-0.

(21) Liu, D.; Mahmood, A.; Weng, D.; Wang, J. Life-Like Motion of Oil Drops at the Air-Liquid Interface. Langmuir 2019, 35 (49), 16146-16152. https://doi.org/10.1021/acs.langmuir.9b02587.

(22) Mokbel, M.; Schwarzenberger, K.; Aland, S.; Eckert, K. Information Transmission by MarangoniDriven Relaxation Oscillations at Droplets. Soft Matter 2018, 14 (45), 9250-9262.

https://doi.org/10.1039/c8sm01720d.

(23) Lagzi, I.; Soh, S.; Wesson, P. J.; Browne, K. P.; Grzybowski, B. A. Maze Solving by Chemotactic Droplets. J. Am. Chem. Soc. 2010, 132 (4), 1198-1199. https://doi.org/10.1021/ja9076793.

(24) Čejková, J.; Novák, M.; Štěpánek, F.; Hanczyc, M. M. Dynamics of Chemotactic Droplets in Salt Concentration Gradients. Langmuir 2014, 30 (40), 11937-11944. https://doi.org/10.1021/la502624f.

(25) Seemann, R.; Fleury, J. B.; Maass, C. C. Self-Propelled Droplets. Eur. Phys. J. Spec. Top. 2016, 225 (11-12), 2227-2240. https://doi.org/10.1140/epjst/e2016-60061-7.

(26) Zöttl, A.; Stark, H. Emergent Behavior in Active Colloids. J. Phys. Condens. Matter 2016, 28 (25), 253001. https://doi.org/10.1088/0953-8984/28/25/253001.

(27) Nguindjel, A.-D. C.; Korevaar, P. A. Self-Sustained Marangoni Flows Driven by Chemical Reactions. ChemSystemsChem 2021, 3, e2100021. https://doi.org/10.1002/syst.202100021.

(28) Babu, D.; Scanes, R. J. H.; Plamont, R.; Ryabchun, A.; Lancia, F.; Kudernac, T.; Fletcher, S. P.; Katsonis, N. Acceleration of Lipid Reproduction by Emergence of Microscopic Motion. Nat. Commun. 2021, 12 (1), 1-7. https://doi.org/10.1038/s41467-021-23022-1.

(29) Hocking, L. M.; Rivers, A. D. The Spreading of a Drop by Capillary Action. J. Fluid Mech. 1982, 121, 425-442. https://doi.org/10.1017/S0022112082001979.

(30) Roman, B.; Bico, J. Elasto-Capillarity: Deforming an Elastic Structure with a Liquid Droplet. J. Phys. Condens. Matter 2010, 22 (49). https://doi.org/10.1088/0953-8984/22/49/493101.

(31) Prasath, S. G.; Marthelot, J.; Menon, N.; Govindarajan, R. Wetting and Wrapping of a Floating Droplet by a Thin Elastic Filament. Soft Matter 2021, 17 (6), 1497-1504. https://doi.org/10.1039/d0sm01863e.

(32) Pokroy, B.; Kang, S. H.; Mahadevan, L.; Aizenberg, J. Self-Organization of a Mesoscale Bristle into Ordered, Hierarchical Helical Assemblies. Science 2009, 323 (5911), 237-240.

https://doi.org/10.1126/science.1165607.

(33) Wu, D.; Chen, Q. D.; Xu, B. Bin; Jiao, J.; Xu, Y.; Xia, H.; Sun, H. B. Self-Organization of Polymer Nanoneedles into Large-Area Ordered Flowerlike Arrays. Appl. Phys. Lett. 2009, 95 (9), 2007-2010. https://doi.org/10.1063/1.3213394.

(34) Bowden, N. B.; Weck, M.; Choi, I. S.; Whitesides, G. M. Molecule-Mimetic Chemistry and Mesoscale Self-Assembly. Acc. Chem. Res. 2001, 34 (3), 231-238. https://doi.org/10.1021/ar0000760.

(35) Vinay, T. V.; Varanakkottu, S. N. Separation of Floating Oil Drops Based on Drop-Liquid Substrate Interfacial Tension. Langmuir 2019, 35 (32), 10596-10600.

https://doi.org/10.1021/acs.langmuir.9b01829.

(36) Vella, D.; Mahadevan, L. The “ Cheerios Effect .” Am. J. Phys. 2005, 73 (9), 817-825. https://doi.org/10.1119/1.1898523.

(37) Hu, Z.; Fang, W.; Li, Q.; Feng, X. Q.; Lv, J. Optocapillarity-Driven Assembly and Reconfiguration of Liquid Crystal Polymer Actuators. Nat. Commun. 2020, 11, 5780. https://doi.org/10.1038/s41467-02019522-1.

(38) Wang, W.; Giltinan, J.; Zakharchenko, S.; Sitti, M. Dynamic and Programmable Self-Assembly of Micro-Rafts at the Air-Water Interface. Sci. Adv. 2017, 3 (5), 1-10.

https://doi.org/10.1126/sciadv.1602522.

(39) Moerman, P. G.; Moyses, H. W.; Van Der Wee, E. B.; Grier, D. G.; Van Blaaderen, A.; Kegel, W. K.; Groenewold, J.; Brujic, J. Solute-Mediated Interactions between Active Droplets. Phys. Rev. E 2017, 96 (3), 1-8. https://doi.org/10.1103/PhysRevE.96.032607.

(40) Arya, P.; Feldmann, D.; Kopyshev, A.; Lomadze, N.; Santer, S. Light Driven Guided and SelfOrganized Motion of Mesoporous Colloidal Particles. Soft Matter 2020, 16 (5), 1148-1155. https://doi.org/10.1039/c9sm02068c.

(41) van der Weijden, A.; Winkens, M.; Schoenmakers, S. M. C.; Huck, W. T. S.; Korevaar, P. A. Autonomous Mesoscale Positioning Emerging from Myelin Filament Self-Organization and Marangoni Flows. Nat. Commun. 2020, 11, 4800. https://doi.org/10.1038/s41467-020-18555-w.

(42) Zou, L. N. Myelin Figures: The Buckling and Flow of Wet Soap. Phys. Rev. E 2009, 79 (6), 061502. https://doi.org/10.1103/PhysRevE.79.061502.

(43) Reissig, L.; Fairhurst, D. J.; Leng, J.; Cates, M. E.; Mount, A. R.; Egelhaaf, S. U. Three-Dimensional Structure and Growth of Myelins. Langmuir 2010, 26 (19), 15192-15199. 
https://doi.org/10.1021/la102726r.

(44) Zou, L. N.; Nagel, S. R. Stability and Growth of Single Myelin Figures. Phys. Rev. Lett. 2006, 96 (13), 138301. https://doi.org/10.1103/PhysRevLett.96.138301.

(45) Elsesser, M. T.; Hollingsworth, A. D. Revisiting the Synthesis of a Well-Known Comb-Graft Copolymer Stabilizer and Its Application to the Dispersion Polymerization of Poly(Methyl Methacrylate) in Organic Media. Langmuir 2010, 26 (23), 17989-17996. https://doi.org/10.1021/la1034917.

(46) Antl, L.; Goodwin, J. W.; Hill, R. D.; Ottewill, R. H.; Owens, S. M.; Papworth, S. The Preparation of Poly(Methyl Methacrylate) Latices in Non-Aqueous Media. Colloids and Surfaces 1986, 17, 67-78. https://doi.org/10.1016/0166-6622(86)80187-1.

(47) Thielicke, W.; Stamhuis, E. J. PIVlab - Towards User-Friendly, Affordable and Accurate Digital Particle Image Velocimetry in MATLAB. J. Open Res. Softw. 2014, 2, e30. https://doi.org/10.5334/jors.bl.

(48) Mele, S.; Söderman, O.; Ljusberg-Wahrén, H.; Thuresson, K.; Monduzzi, M.; Nylander, T. Phase Behavior in the Biologically Important Oleic Acid/Sodium Oleate/Water System. Chem. Phys. Lipids 2018, 211 (September 2017), 30-36. https://doi.org/10.1016/j.chemphyslip.2017.11.017.

(49) Soh, S.; Bishop, K. J. M.; Grzybowski, B. A. Dynamic Self-Assembly in Ensembles of Camphor Boats. J. Phys. Chem. B 2008, 112 (35), 10848-10853. https://doi.org/10.1021/jp7111457. 